\title{
Identical STEMI Pattern in Two Siblings with Different Risk Factors
}

\author{
Renata Gerculy ${ }^{1}$, Camelia Libenciuc ${ }^{1}$, Nora Rat ${ }^{1,2}$, Istvan Kovacs ${ }^{1,2}$, Monica Chitu ${ }^{1,2}$, \\ Imre Benedek ${ }^{1,2}$, Theodora Benedek ${ }^{1,2}$ \\ ${ }^{1}$ Clinic of Cardiology, Emergency Clinical County Hospital, Târgu Mureș, Romania \\ ${ }^{2}$ Center of Advanced Research in Multimodality Cardiac Imaging, CardioMed Medical Center, Târgu Mureș, Romania
}

\begin{abstract}
Environmental factors may have an important role in the development of coronary heart disease. However, it is not clearly understood yet how the genetic factors interplay with the environmental ones in the onset of acute myocardial infarction. The early onset of coronary artery disease in cases with a positive family history suggests a certain role of genetic predisposition, but the open question remains: could environmental differences contribute to this predisposition? This case report describes similar coronary angiographic findings of two brothers who developed acute myocardial infarction in their early 40s, after being exposed to different environmental risk factors.
\end{abstract}

Keywords: siblings, coronary artery disease, genetic predisposition, ankylosing spondylitis

\section{ARTICLE HISTORY}

Received: July 20, 2020

Accepted: August 27, 2020

\section{CORRESPONDENCE}

\section{Camelia Libenciuc}

Str. Gheorghe Marinescu nr. 50 540136 Târgu Mureș, Romania Tel: +40 265212111

E-mail: camelialibenciuc@gmail.com

\section{INTRODUCTION}

Coronary artery disease (CAD) is one of the leading global causes of mortality and morbidity. The influence of genetic and environmental factors on CAD has been of great interest for researchers since the 1950s. We present the case of two brothers who developed acute myocardial infarction in their early 40s, with nearly identic coronary artery involvement and the same localization of the culprit lesion. The most relevant difference between the siblings was that one of the brothers was diagnosed with an autoimmune condition - ankylosing spondylitis (AS), which was associated with permanent systemic inflammation, putting him at a higher risk for myocardial infarction. The coronary lesions found in his sibling, who presented the same clinical pattern of an acute myocardial infarction, were similar, thus suggesting the role of genetic predisposition for coronary heart disease. In addition, the patients presented a positive family history of early coronary artery disease.

\section{CASE REPORT}

Brother A is a 45-year-old man, with a body mass index of $35.3 \mathrm{~kg} / \mathrm{m}^{2}$, a medical history of hypertension, hypercholesterolemia, and medically controlled (with biological therapy) AS (Bechterew's disease). The patient presented to the emergency department (ED) of a secondary care 

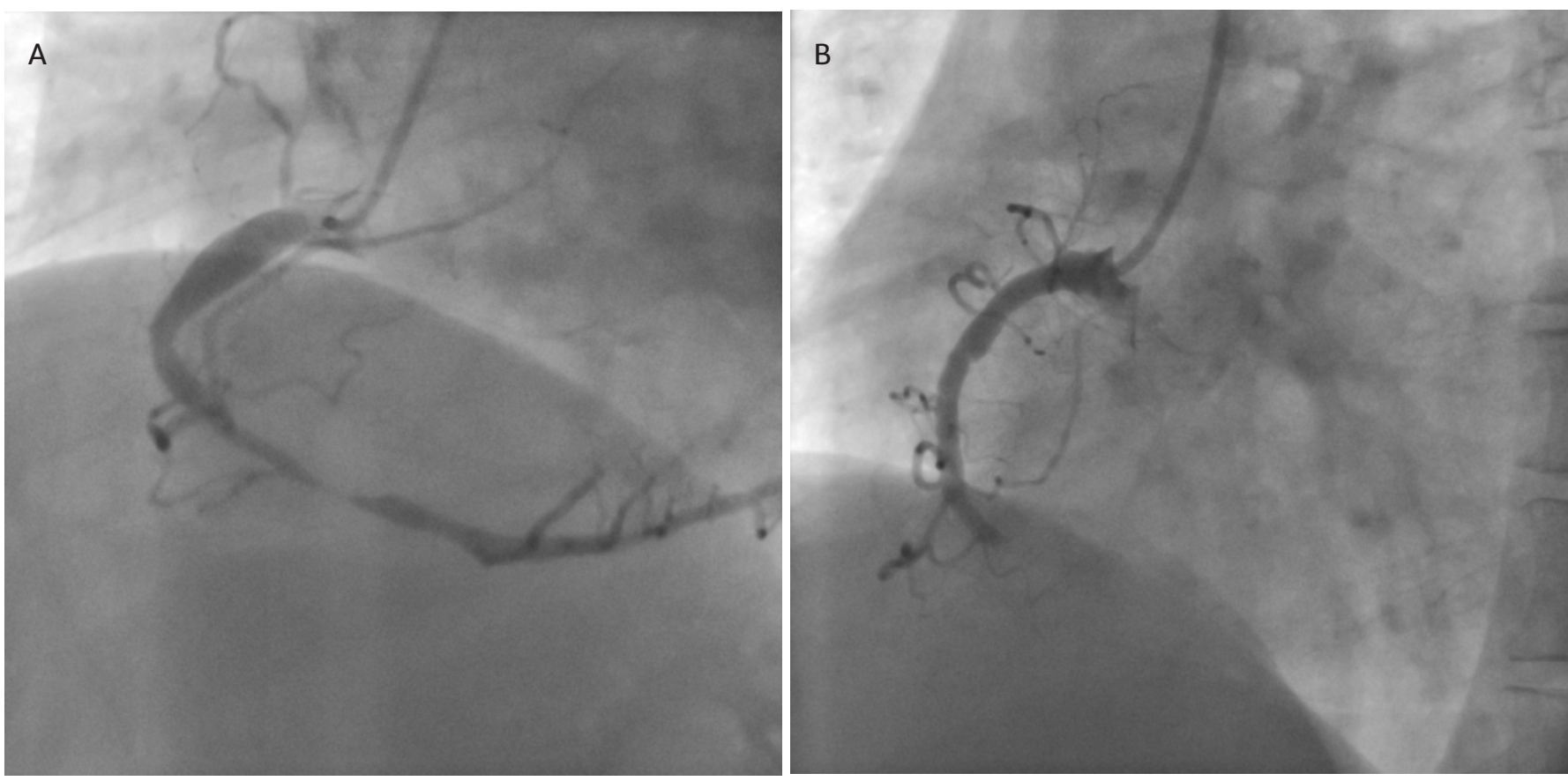

FIGURE 1. Invasive coronary angiography revealing the right coronary artery with significant stenoses in brother $A(A)$ and brother $B$ (B)

hospital, complaining of typical acute chest pain with radiation in the left upper limb, associated with shortness of breath. The 12-lead electrocardiogram (ECG) revealed a $2-3 \mathrm{~mm}$ elevation of the ST segment in leads DII, DIII, and aVF, as well as ST depression in DI and aVL. Findings on the physical examination and blood tests were unre- markable, except for a moderate increase in cardiac enzymes. Transthoracic echocardiography showed discrete hypokinesis of the inferior wall of the left ventricle. As the clinical presentation and ECG changes were suggestive for the diagnosis of ST-segment elevation acute myocardial infarction (STEMI) of the inferior and lateral wall of the
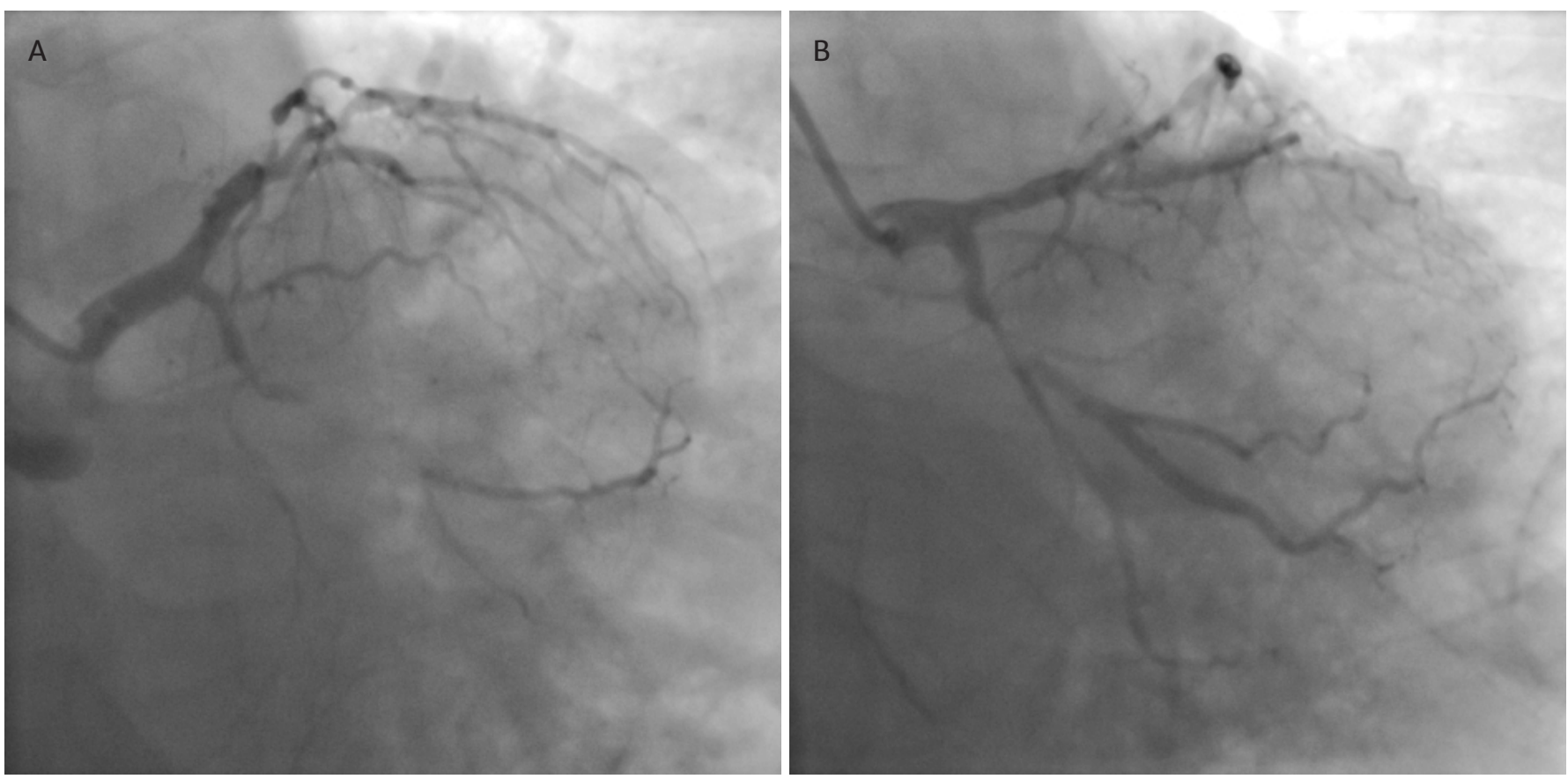

FIGURE 2. Invasive coronary angiography revealing the left coronary artery with significant stenoses of the anterior descending artery and circumflex artery, in brother $\mathrm{A}(\mathbf{A})$ and brother B (B) 
left ventricle, the patient was immediately transferred to a tertiary care hospital with a cardiac catheterization laboratory. The emergency coronary angiography revealed right coronary artery (RCA) domination, with a severe stenosis in the vertical segment (Figure 1A). The left coronary artery presented a moderate $50 \%$ stenosis in the distal segment, the first diagonal artery had a severe stenosis of up to $80 \%$, while the circumflex artery presented a severe stenosis in the middle segment (Figure 2A). The culprit lesion was considered the one located at the level of the RCA and was treated by primary PCI with stent implantation. The patient was stable during and after the procedure, and the revascularization procedure was considered successful.

Brother B is a 43-year-old man with a body mass index of $22.9 \mathrm{~kg} / \mathrm{m}^{2}$, chronic smoker, without any other comorbidities, who presented to the emergency room less than one year after his brother had been diagnosed with an acute coronary syndrome. At presentation, he complained of similar symptoms, with acute onset of constrictive chest pain with dyspnea. The symptoms had started 6 hours before presentation. The physical examination revealed normal heart and lung sounds, while the laboratory tests found elevated levels of cardiac troponins and $\mathrm{CK}-\mathrm{MB}$, and elevated total cholesterol levels $(200 \mathrm{mg} /$ dL). The 12-lead ECG revealed ST-segment elevation of more than $2 \mathrm{~mm}$ in the inferior leads, with concordant ST-segment depression, and the transthoracic echocardiography showed discrete hypokinesis of the inferior and posterior wall of the left ventricle. The patient underwent emergency coronary angiography for inferior STEMI, which revealed atherosclerotic infiltration of the left anterior descending artery (LAD) with a 70\% stenosis in the middle segment. The circumflex artery presented a diffuse atherosclerotic involvement with a significant coronary lesion in the proximal segment, with $80 \%$ stenosis (Figure 2B). The RCA presented an acute occlusion in the vertical segment (Figure $1 \mathrm{~B}$ ), which was treated by primary percutaneous coronary angioplasty with drug-eluting stent. A TIMI 3 flow was recorded after the procedure. The procedure was uneventful, and the patient was monitored in the intensive cardiac care unit for 48 hours, receiving optimal medical treatment and was discharged after 5 days of hospitalization. The patients agreed to the publication of their data, and the institution where the patients had been admitted, approved the publication of the cases.

\section{DISCUSSIONS}

There are several points of interest in the two cases: (1) first-degree relatives with almost the same age at the on- set of the myocardial infarction; (2) relatively young age of CAD diagnosis; (3) similar coronary artery involvement; (4) same localization of the culprit lesion, in the right coronary artery; (5) persistent inflammatory status; (5) positive history of CAD on the maternal side; (6) elevated blood cholesterol levels in both brothers.

The hereditary nature of CAD is well known, but the most remarkable observation in these cases is the different predisposing cardiovascular risk profiles of the two brothers. Brother A had a history of a chronic systemic autoimmune disease. AS is a rare condition that primarily affects the sacroiliac joints and the axial skeleton, but can also cause extra-articular complications involving the heart, lungs, eyes, and nervous system.

Several studies have investigated the existence of increased cardiovascular risk in chronic inflammatory rheumatic diseases, especially in AS. The persistently elevated chronic inflammatory response is considered a non-traditional risk factor for CAD and has been associated with accelerated atherosclerosis. ${ }^{1-5}$ The underlying inflammatory process in AS plays a pivotal role in developing CAD by promoting a hypercoagulable state due to excessive activation of the coagulation cascade and inhibiting the anti-coagulation and fibrinolytic pathway. ${ }^{5}$ Afterwards, the oxidative stress and inflammatory cytokines are also contributing to the development of premature CAD by promoting endothelial dysfunction. In addition, patients with AS have an increased risk of developing metabolic syndrome and dyslipidemia. Also, a considerable increase in intima-media thickness was found in AS, which is a marker of subclinical atherosclerosis. ${ }^{1,3}$ Besides the inflammatory effects of AS on the endothelium and the accelerated atherosclerotic process, the limited functional capacity due to chronic joint pain leads to sedentary lifestyle and weight gain, increasing cardiovascular risk and favoring the development and vulnerabilization of coronary atherosclerosis. ${ }^{6}$ The average prevalence rate of CAD in AS patients is about two- to three-fold higher in comparison with the general population. ${ }^{1,2}$ Nonetheless, brother B developed an acute myocardial infarction even without this highly predisposing risk factor, at almost the same age as his brother, with an identical coronary artery involvement.

Families with clustering of coronary heart disease represent $14 \%$ of the community, but account for $72 \%$ of young affected patients, which reflects shared behavioral, environmental, and genetic factors within families. Furthermore, a history of CAD in a first-degree family member is associated with a 1.5-3-fold increased risk of the disease. Accordingly, the risk may be increased more than 10 times if a brother is affected before the age of 45 years. 
Also, the risk increases with the number of affected family members, especially from an early age. ${ }^{7}$

The most recent studies have estimated approximately 60 genetic loci linked to coronary risk. The role of genetic mutations in $\mathrm{CAD}$ was discovered in cases with familial aggregation of coronary atherosclerosis. The most common cause of family clustering of CAD is familial hypercholesterolemia. It is considered the primary monogenic driver of $\mathrm{CAD}$, leading to accelerated atherosclerosis. ${ }^{8}$ In both cases, high blood cholesterol levels were identified, which could explain the development of CAD at this relatively young age, possibly due to accelerated atherosclerosis.

Maurovich et al., in a large prospective heritability study, have described atherosclerosis as a geometrically focal disease with the capacity of involving the inner curvature of coronary vessels and the outer edges of their bifurcations. ${ }^{9}$ These areas are considered susceptible due to slower blood flow and direction changing of the blood with the cardiac cycle. This results in a low hemodynamic shear stress, which may cause endothelial damage. Furthermore, coronary vascular areas with a low variability of hemodynamic shear stress are less affected by atherosclerosis. Therefore, the geometry of coronary arteries should be considered a potential risk factor for the development of atherosclerosis. ${ }^{8}$ However, there is large variation in the anatomical configuration of coronary arteries from individual to individual, and there are no evident data about the genetic and environmental influences in the development of coronary artery geometry.

\section{CONCLUSIONS}

The importance of gene-environment interaction is a highly investigated subject in cardiovascular research, but it has not been yet elucidated how these interactions act in the complex development of cardiovascular diseases. The particularity of the presented cases is that one brother had an underlying chronic inflammatory disease, while the other presented modifiable cardiovascular risk factors (smoking). Despite this, both brothers presented an acute inferior STEMI, with the same location of the infarct-related artery and similar associated coronary lesions in the non-culprit coronary branches. At the same time, both brothers required coronary stent angioplasty at roughly the same age (early 40s). This interesting clinical scenario brings to light the relationship between genetic and environmental factors in the development of coronary atherosclerosis and acute cardiovascular events. More likely, the elevated cholesterol levels associated with familial hypercholesterolemia and the positive family history suggest the high impact of genetic predisposition for developing myocardial infarction. The genetic risk factors are not modifiable, but efforts should be made in the area of gene sequencing and phenotyping, in order to identify more suitable prevention strategies and future treatments.

\section{CONFLICT OF INTEREST}

Nothing to declare.

\section{ACKNOWLEDGEMENT}

This research was supported via the research grant no. 103544/2016, contract number 26/01.09.2016, entitled "Increasing the research capacity in the field of vulnerable plaque imaging, based on advanced nanoparticles, fusion imaging and computational simulation - PlaqueImage", financed by the Romanian Ministry of European Funds, the Romanian Government and the European Union.

\section{REFERENCES}

1. Park CJ, Choi YJ, Kim JG, et al. Association of Acute Myocardial Infarction with ankylosing Spondylitis: A nationwide longitudinal cohort study. J Clin Neurosci. 2018;56:34-37. doi: 10.1016/j.jocn.2018.08.008.

2. Peters MJ, Visman I, Nielen MM, et al. Ankylosing spondylitis: a risk factor for myocardial infarction?. Ann Rheum Dis. 2010;69:579-581. doi: 10.1136/ard.2009.110593.

3. Mathieu S, Gossec L, Dougados M, Soubrier M. Cardiovascular profile in ankylosing spondylitis: a systematic review and meta-analysis. Arthritis Care Res (Hoboken). 2011;63:557563. doi: 10.1002/acr.20364.

4. Mathieu S, Pereira B, Soubrier M. Cardiovascular events in ankylosing spondylitis: an updated meta-analysis. Semin Arthritis Rheum. 2015;44:551-555. doi: 10.1016/j. semarthrit.2014.10.007.

5. Ungprasert P, Srivali N, Kittanamongkolchai W. Risk of coronary artery disease in patients with ankylosing spondylitis: a systematic review and meta-analysis. Ann Transl Med. 2015;3:51. doi: 10.3978/j.issn.2305-5839.2015.02.05.

6. Christiansen MK. Early-onset Coronary Artery Disease Clinical and Hereditary Aspects. Dan Med J. 2017;64:B5406.

7. Khera AV, Kathiresan S. Genetics of coronary artery disease: discovery, biology and clinical translation. Nat Rev Genet. 2017;18:331-344. doi: 10.1038/nrg.2016.160.

8. Scheuner MT. Genetic evaluation for coronary artery disease. Genet Med. 2003;5:269-285. doi: 10.1097/01. GIM.0000079364.98247.26.

9. Maurovich-Horvat P, Tárnoki DL, Tárnoki ÁD, et al. Rationale, Design, and Methodological Aspects of the BUDAPESTGLOBAL Study (Burden of Atherosclerotic Plaques Study in Twins-Genetic Loci and the Burden of Atherosclerotic Lesions). Clin Cardiol. 2015;38:699-707. doi: 10.1002/clc.22482. 